\title{
Horizons/Théâtre
}

Revue d'études théâtrales

13 | 2018

Les arts du spectacle dans l'Afrique subsaharienne - 1

\section{Le constituant négro-africain dans le théâtre moderne}

\section{Azouine Abdelmajid}

\section{(2) OpenEdition}

1 Journals

Édition électronique

URL : https://journals.openedition.org/ht/1012

DOI : $10.4000 /$ ht. 1012

ISSN : 2678-5420

Éditeur

Presses universitaires de Bordeaux

\section{Édition imprimée}

Date de publication : 31 décembre 2018

Pagination : 68-76

ISBN : 979-10-300-0318-5

ISSN : 2261-4591

\section{Référence électronique}

Azouine Abdelmajid, "Le constituant négro-africain dans le théâtre moderne », Horizons/Théâtre [En ligne], 13 | 2018, mis en ligne le 01 janvier 2019, consulté le 17 mai 2022. URL : http://

journals.openedition.org/ht/1012 ; DOI : https://doi.org/10.4000/ht.1012

\section{(c) $(1) \odot$}

La revue Horizons/Théâtre est mise à disposition selon les termes de la Licence Creative Commons Attribution - Pas d'Utilisation Commerciale - Pas de Modification 4.0 International. 


\section{AZOUINe ABdelmajid}

Abdelmaiid Azouine est docteur en Lettres et Sciences humaines, enseignant de théâtre et d'histoire de l'art à la faculté des Lettres et des Sciences humaines, Université Mohammed V, Rabat, et membre permanent au sein du laboratoire de recherche "Langues, Littératures, Arts et cultures (L.L.A.C.) » (département Études françaises, FLSH, U. Med V, Rabat).

Il a publié entre autres: Théâtre moderne et pratiques picturales, correspondances et confluences (L'Harmattan, 2015); "Le spectacle théâtral et ses doubles, l'exemple du "théâtre peint" de Jean Dubuffet », Plasticité, n० 1, juillet 2015; "L'art brut est-il un anti-art? », actes du colloque international "Les valeurs éthique et esthétique dans la littérature et les arts », ENS, Université Mohammed V, Rabat, 2016.

Mail: majid6023@hotmail.com

Résumé : La tentative de désoccidentaliser le théâtre au lendemain de la naissance de la tendance avant-gardiste au théâtre a entraîné une certaine ouverture de la littérature théâtrale sur d'autres cultures et sur d'autres traditions extra-européennes en l'occurrence celle africaine. Le constat puise sa légitimité tant sur le plan des théories et des écrits sur le théâtre que sur le plan la mise en scène et essentiellement de la dramaturgie. Il suffit dans ce sens de rappeler qu'Antonin Artaud dans sa conception du théâtre de la cruauté prône un retour aux origines rituelles du jeu théâtral, que certains dramaturges ont fait de la cause des noirs et des marginaux leurs thèmes de prédilections.

Cet article interroge dans ce sens le retour du jeu masqué et du rite, la reconstruction de l'espace scénique et la réexploitation de l'objet dans certaines formes théâtrales modernes comme le happening, le théâtre de participation ef la performance. Dans un second temps, il met l'accent sur les figures du noir, de l'africain asservi chez Jean Genet à travers sa pièce Les Nègres.

Mots-cLÉs : théâtre moderne, cérémonial, rite africain, jeu masqué, mise en scène, dramaturgie, esthétique.
ABstract :It seems clear that the attempt to withdraw the western character of the theater after the birth of the avant-garde fendency of the theater led to a certain opening of the theatrical literature to other cultures and other traditions outside Europe as is the case for Africa. The note finds its legitimacy in terms of theories and writings on stage and in terms of graduation, especially dramatic subjects. In this sense, it suffices to recall, for example, that Antonin Artaud in his conception of the theater of cruelty calls for a return to the ritual origins of the theatrical scene, which made certain black and marginalized playwrights in their favorite subjects.

We intend to question in this sense, as a first step, about the return of the masked game and the rite in certain modern theatrical forms such as happening, participation theater, and performance. In a second step, we will focus on the figures of black with Jean Genet through his scene Les Nègres The Blacks.

KeYwords: modern theater, ceremonial, african rite, masked game, staging, dramaturgy, aesthetics. 


\section{Le constituant négro-africain dans le théâtre moderne}

IL PARAÎT ÉVIDENT QUE LA TENTATIVE DE DÉSOCCIDENTALISER le théâtre au lendemain de la naissance de la tendance avant-gardiste au théâtre a entraîné une certaine ouverture de la littérature théâtrale sur d'autres cultures et sur d'autres traditions extra-européennes en l'occurrence celle africaine. Le constat puise sa légitimité tant sur le plan des théories et des écrits sur le théâtre que sur le plan de la mise en scène et essentiellement de la dramaturgie. Il suffit dans ce sens de rappeler qu'Antonin Artaud dans sa conception du théâtre de la cruauté prône un retour aux origines rituelles du jeu théâtral, que certains dramaturges ont fait de la cause des noirs et des marginaux leurs thèmes de prédilection.

Nous comptons interroger dans ce sens, le retour du jeu masqué et du rite dans certaines formes théâtrales modernes comme le happening, le théâtre de participation, et la performance. Dans un second temps, nous mettrons l'accent sur les figures du noir chez Jean Genet à travers sa pièce Les nègres.

Dès le début du $\mathrm{xx}^{\mathrm{e}}$ siècle, nombreuses sont les inquiétudes esthétiques qui ont suscité l'intérêt des praticiens du théâtre : il s'agit tout d'abord de se démarquer de l'esthétique d'un théâtre bourgeois d'obédience naturaliste connu par ses thèmes et fidèle à ses traditions héritées du drame. La rupture était ainsi par rapport à une dramaturgie « de grande consommation » telle qu'elle a été taxée par Brecht. Cette rupture va conduire le théâtre sur les voies de l'expérimentation qui donnera naissance à un théâtre à vocation avantgardiste, à même d'assouvir les envies rénovatrices des dramaturges.

De là, certaines traditions relevant de l'identité et de la culture africaine seront convoquées pour prêter main-forte à cette nouvelle dramaturgie naissante. Nous faisons ici allusion à titre indicatif et non limitatif au masque, au retour du rituel, du cérémonial, du carnavalesque, et la célébration de la parole dans l'événement théâtral.

En ce qui concerne le masque, les historiens du théâtre sont unanimes quant à ses premières exploitations dans les scènes qui remontent au théâtre 
grec et à la commedia dell'arte, avant qu'il ne connaisse une sorte de déclin dûe à l'hégémonie de l'esthétique naturaliste. Celle-ci stipule que le jeu masqué affecte l'aspect véridique de l'acte théâtral, et va à l'encontre de la théâtralité qu'elle veut dissimuler. Il connaitra une sorte de regain dans le théâtre contemporain, notamment au début $\mathrm{du} \mathrm{xx}^{\mathrm{e}}$ siècle, lorsque la plupart des fondements dramaturgiques, entre autres le personnage, ont été remis en cause. Le masque correspond ainsi à une phase où la portée psychologique du personnage est rejetée au profit de l'exaltation du corps et de l'expression corporelle.

Edward Gordon Craig et Antonin Artaud furent les précurseurs de cette esthétique théâtrale qui voit dans le masque un moyen de régénérer la pratique théâtrale. Leurs écrits «l'acteur et la surmarionnette », « à propos des masques $\gg$, et « le théâtre et son double » demeurent des textes fondateurs quant à l'instauration d'une nouvelle tendance théâtrale qui fait du masque un élément incontournable de l'expression dramaturgique.

Pour Craig, la valeur ajoutée que le masque apporte à l'exercice théâtral, c'est qu'il permet à l'artiste une sorte d'ajustement de l'expression avec la situation dramatique, tandis que l'expression du visage recèle quelque chose de superflu et d'inquiétant qui peut nuire à la cohésion de l'événement théâtral. C'est ce qui justifie son parti pris à l'égard du masque: «Je pense, naturellement, que le visage est un pauvre instrument. Argile ou marbre sont meilleurs que la chair lorsque l'œuvre de l'artiste doit y être enchâssée ${ }^{1}$. » Face à l'insuffisance du visage à produire une œuvre de théâtre authentique, du fait que le comédien se trouve toujours sous l'emprise de ses émois, le masque se dresse alors comme le garant de cette authenticité recherchée. C'est pour cette raison que Craig le place comme une condition nécessaire et suffisante pour toute création théâtrale en affirmant:

Au contraire, je dis que nous voudrons réaliser et que nous réaliserons une pièce seulement lorsque nous aurons appris à faire un masque; car le masque et la tête idéale du théâtre; c'est le seul moyen possible pour exprimer de manière juste la forme extérieure et visible de la création du poète ${ }^{2}$.

Quant à Antonin Artaud, dans sa tentation d'en finir avec les chefs-d'œuvre, de rejeter catégoriquement le texte de l'auteur, de dépsychologiser le jeu théâtral, et de vouloir meubler l'espace de la scène, il s'est inspiré des traditions extra-européenne en l'occurrence africaines pour prôner le masque comme outil dramaturgique qui permet à l'acteur de dépasser ses affects et en même temps au spectateur de ne pas se laisser leurrer par l'effet d'identification. 
Les positions de ces deux théoriciens ont eu un retentissement manifeste chez de nombreux metteurs en scène qui commencent à interroger les possibles scéniques offerts par le masque. Ainsi Peter Brook conçoit sa réflexion à partir du masque balinais et africain. Ariane Mnouchkine, de sa part, tente d'explorer dans ses spectacles toutes les potentialités du jeu masqué. Quant à Adolph Appia, il considère que le masque constitue une sorte de dépersonnalisation de l'acteur.

Le masque n'a de sens que dans une situation dramatique, lorsqu'il est chaussé par un comédien, comme le souligne Odette Aslan, en sa qualité d'académicienne spécialiste du jeu de l'acteur et de l'analyse de la mise en scène contemporaine: « Ils (les masques) ne pourraient retrouver leur entière signification qu'animés par la chair et le sang de leurs porteurs, mus par la musique et la danse dont ils procédaient, décodés par les membres d'une société initiée ${ }^{3}$. 》

Si le retour au masque a permis au théâtre moderne de renouer avec ses origines sacrées et festives, il faut aussi reconnaitre que dans sa recherche d'une théâtralité brute, d'une théâtralité qui se crée loin des codes et des connivences longtemps rabâchés par les metteurs en scène, le souci esthétique de désoccidentaliser la pratique théâtrale va naître. Et c'est là où l'Afrique comme lieu ancré dans les traditions ancestrales ou cohabitent souvenir et célébration, rite et solennité, procession majestueuse et initiation sera une terre de recueillement et de pèlerinage pour les metteurs en scène en quête de renouveau. Et dans une influence réciproque, le théâtre africain naissant des années trente connu sous le non de théâtre pontin sera essentiellement culturaliste et folkloriste reproduisant pour une grande part les schèmes de l'idéologie coloniale.

Ce retour à la célébration et au moment théâtral, perçu comme commémoration où regardants et regardés se partagent d'abord un plaisir qui les réunit dans le hic et nunc, donnera naissance à une esthétique novatrice dans la pratique théâtrale. Il s'agit de ce que les critiques appelleront le théâtre de participation ou le théâtre forum, basé foncièrement sur l'ébranlement du quatrième Mur pour reprendre la métaphore brechtienne très éloquente. L'idée est de déconstruire et d'élargir l'ère de jeu en effaçant ainsi toute forme de contours séparant la scène et la salle. Désormais, le présent ambiant de l'événement théâtral devient perméable à tout un chacun pouvant intervenir pour contribuer à son enrichissement. Ceci allait offrir une marge beaucoup plus importante à l'improvisation comme une autre esthétique théâtrale à part entière. À titre illustratif nous pouvons citer dans ce sens le Kotéba comme forme de 
théâtre traditionnel au Mali. Annuellement, après la saison des récoltes, les villageois se réunissaient pour assister à des saynètes mises en place par les jeunes du village. L'objectif est de se moquer des mauvaises conduites et des comportements inappropriés, les comédiens ont une grande marge de liberté dans l'expression mais sans jamais désigner nommément quelqu'un.

Dès lors des rites d'initiation ou de passage et des célébrations seront revisités, des collaborations vont avoir lieu et des esthétiques nouvelles verront le jour : théâtre de participation, happening, et improvisation. Et dans ce retour au rite et à la célébration dans leur acception première, l'espace théâtral et son organisation feront l'objet d'un intérêt particulier par les metteurs en scène et les scénographes. Ces derniers vont opter pour la simplicité voire le dépouillement même de cette aire de jeu comme un choix esthétique doté d'une pléthore de significations. Il s'agit essentiellement d'ancrer l'événement théâtral dans une sorte d'espace vide tel que le conçoit Peter Brook, un espace qui met en relief le potentiel des acteurs et leur présence corporelle et verbale au détriment de tout autre accessoire théâtral. Ce qui pourrait aussi faire allusion à toute l'esthétique de pauvreté telle qu'elle a été théorisée par Jerzy Grotowski dans son ouvrage Vers un théâtre pauvre où il conceptualise cette notion comme suit « nous pouvons donc définir le théâtre comme « ce qui se passe entre spectateur et acteur. Toutes les autres choses sont supplémentaires peut-être nécessaires, mais quand même supplémentaires ${ }^{4} \gg$.

Dans le même esprit, le dépouillement de l'espace théâtral, lorsqu'il procède par un effacement entier de toute sorte de repère spatial a conduit les metteurs en scène et les scénographes à la conception du no man's land, c'està-dire un espace de jeu qui se refuse à toute catégorisation et qui rejette tout ancrage aussi bien temporel que culturel.

Dans le même sens, le retour au rituel et à la célébration, comme il nous a permis de reconstruire l'espace théâtral, nous a aussi, semble-t-il, ouvert le champ d'exploitation de l'objet dans le faire théâtral. Arraché à sa dimension utilitaire habituelle à travers des détournements, des symbolisations, l'objet est intellectualisé, investi dans sa dimension esthétique, ce qui pourrait nous rappeler toute la tendance du théâtre d'objet telle que Tadeusz Kantor l'a conçue.

En rapport avec l'esprit de remise en question des traditions théâtrales précédemment évoqué, le statut du texte, longtemps célébré, au sein de l'exercice dramatique a été réexaminé voire désacralisé. Son règne absolu se trouve donc voué à l'échec et même les métiers qui étaient toujours à son service, en l'occurrence le scénographe et le décorateur, vont revendiquer leur droit 
à l'autonomie. De même les précurseurs de cette nouvelle tendance dans le théâtre réclameront qu' « il est temps de détrôner sir le mot ! ${ }^{5} \gg$.

La vision d'Antonin Artaud, dans ce sens, constitue l'une des expériences phares. Son ouvrage Le théâtre et son double est considéré comme étant le cadre théorique d'une nouvelle théâtralité décloisonnée de la tutelle du textuel et du signifié, où le texte aura un autre rôle à assumer: il n'est plus le véhicule d'une quelconque charge intellectuelle, mais, à travers son pouvoir incantatoire, il devient le lieu et le moyen d'un ébranlement cathartique du spectateur. C'est pourquoi, Artaud a placé cette entité théâtrale au centre de ses recherches quant à la définition d'une nouvelle esthétique théâtrale. Sa démarche consiste à $: \ll$ Briser le langage pour toucher la vie, c'est faire ou refaire le théâtre ${ }^{6} \gg$.

Ainsi le concept d'un théâtre dit de la parole prendra la place de ce théâtre qui a toujours considéré le mot littéraire comme point de départ, c'est un théâtre qui va substituer à la matérialité du texte la consistance de l'oralité. De là, le recours à des cultures de traditions orales comme les traditions africaines. Le griot africain, ce sac à parole, sans lequel des pratiques séculaires auraient disparu sera une source d'inspiration et un modèle à imiter. La parole marquera ce passage vers un théâtre où la dramatisation se base, de surcroît sur une parole retentissante et plus fonctionnelle qui vient en appui à l'action, une parole ayant son poids et son écho dans la progression dramatique de l'intrigue.

En ce qui concerne le cheminement évolutif que le théâtre africain a traversé, une première phase de naissance et de positionnement par rapport aux autres genres demeure incontournable. Ainsi, il va puiser dans un fond identitaire et culturel propre à l'Afrique comme terre de traditions et de pratiques sociales et tribales séculaires. Et lorsque ce théâtre a décidé de rompre avec sa première vocation culturaliste et folkloriste qui l'a marqué pendant l'époque coloniale, il va verser dans la réhabilitation de tout ce qui renvoie à l'identité africaine, comme dans une tentative de la part des dramaturges africains d'assumer une part de la revendication de la cause des noirs dans une humeur de contestation et de prise de conscience à l'image de ce que la poésie de la négritude a accompli. Ainsi théâtre africain et tendance épique brechtienne tissent des affinités et une vision fonctionnelle de l'événement théâtral au-delà de toute dimension spectaculaire exhibitionniste simplette. C'est ce qui revient sous la plume de Mario de Andrade Dans la préface de Continent-Afrique de Nenekhaly-Camara, il reconnaît que: «Dans la lutte qu'ils mènent pour bâtir leur propre avenir, les peuples africains ont besoin 
de se reconnaître dans leurs héros du passé. Ceux-là mêmes qui, ayant résisté avec les moyens limités de leur temps à la conquête coloniale, sont considérés par les générations modernes comme les phares de l'espérance. Voilà pourquoi le théâtre épique vient à son heure ${ }^{7}$. $\gg$ Un autre exemple assez significatif est L'exil d'Albouri de Cheik Aliou Ndao (1967) qui met en scène Ali Bouri Ndiaye, roi du Djolof, ce dernier incarne l'image du guerrier indomptable, il s'oppose à la domination française avec une intrépidité folle. Et à la fin il préfère l'exil avec tout son peuple à la soumission - en poursuivant avec l'énergie du désespoir un combat qui ne finit qu’à sa mort.

Nous constatons à travers ces deux exemples à quel point le théâtre africain a assumé la cause du noir colonisé et surexploité par l'Occidental, comment la scène était une tribune pour exprimer la résistance et le refus de tout projet d'acculturation, ce qui va nous conduire à examiner comment cette même thématique sera abordée par une figure emblématique du nouveau théâtre qui est Jean Genet à travers sa pièce Les nègres.

La question provocatrice qui a amené Jean Genet à écrire cette pièce est la suivante: «Mais qu'est ce qu'un noir et, c'est donc de quelle couleur? » L'objectif premier était d'aborder cette rupture tragique entre le Blanc et le Noir, dans un souci de construire une imagerie en parfait écart avec les représentations courantes spéculées autour du Noir.

Le choix d'abord du titre « les nègres » est très révélateur dans la mesure où il trace une ligne de démarcation claire entre l'être authentique, le Noir, et le nègre an tant que pure invention du Blanc. Donc si l'histoire met en jeu des nègres jouant le procès d'un crime d'une jeune femme blanche par l'un d'entre eux devant une Cour allégorique de Blancs, ce procès est en fait celui des Blancs contre leur propre invention. Le jugement donc simulé sur scène permet une certaine revanche dans le sens où il opère par une sorte d'inversement de rôles où l'oppresseur devient opprimé, le juge devient coupable et celui qui a longtemps confisqué au Noir son droit à la parole doit l'écouter.

Il faut, de prime abord, signaler que les noms des nègres ne portent aucune mention patronymique ou matronymique exemple: Archibald Absalon Wellington, Edgard-hélas Ville de Saint-Nazaire. La figure du nègre est donc assimilée à un être déterritorialisé, déraciné de sa culture et de son identité déjà à travers un système onomastique outrageux qui le force à taire son nom africain et à vivre dans une sorte d'exil nominal. Ce qui est révélé par Archibald: «Menteurs, les noms que je vous ai livrés sont faux ${ }^{8}$. »Leur fausseté tient donc au fait qu'ils sont une pure désignation émanant de l'Autre, le Blanc, sans aucun lien avec l'être du Noir. Ne serait-ce que pour marquer 
une certaine appropriation esclavagiste, une autre manière de dénoncer voire d'actualiser scéniquement la pratique humiliante de l'étampage qui a longtemps offusqué l'être noir, mais cette fois-ci, en nommant.

L'une des facettes du Noir est traduite scéniquement à travers un jeu emboîté, les comédiens seront amenés à transposer sur scène, et en termes de couleur, les rapports de force, les contradictions, les revendications qui marquent leurs rapports aux Blancs; ainsi la dichotomie Noir/Blanc trouve une parfaite illustration au niveau du jeu dans le jeu. Les nègres feignent ce qu'ils ne sont pas et finissent par entrer dans leur destinée, une destinée façonnée par leur opposant ontologique et historique le Blanc: ils s'assimilent ainsi à une figure fantomatique, à un être répugnant, barbare et sanguinaire lié au viol, au meurtre et à la violence. Archibald nous fait apprendre dans ce sens :

Que les Nègres s'obstinent jusqu'à la folie dans ce qu'on les condamne à être, dans leur ébène, dans leur odeur, dans leur jaune, dans leurs goûts cannibales?.

Le jeu dans le jeu revêt plusieurs pelures; il use du dit et du montré, du scénique et du hors-scénique, du déguisement et du dévoilement. Toute cette prolixité est mise au service de la dénonciation de l'oppression, des clichés et des connotations outrageantes dont souffrait le Noir. Cette mise en abîme ou ce méta-jeu s'exacerbe dans une sorte de frénésie lorsque les comédiens se démasquent en se débarrassant de leur deuxième peau blanche après l'exécution du coupable noir dans les coulisses.

Donc, au-delà de son rôle en n'étant qu'un accessoire scénique et un avatar du simulacre, le masque est un outil qui permet au Noir de passer de l'univers des Noirs vers celui des Blancs assurant ainsi un certain compromis entre les deux univers antagonistes. Il constitue à cet effet un renvoi direct au double, à la tyrannie du paraître sur l'être, que doit endosser le Noir pour s'assimiler au nègre ou au blanchi. Cependant, il faut admettre que le fait d'accepter le port du masque lui permet dans une certaine mesure de résister et de se protéger contre sa dépossession identitaire par le Blanc.

L'importance attribuée au jeu théâtral dans la construction d'une nouvelle imagerie autour du noir tient au fait qu'il est la seule issue qui se profile devant cet être longtemps écrasé par diverses formes d'injustice et anéanti par sa condition subalterne comme l'atteste Archibald: « Le seul domaine qui nous reste, Le théâtre ${ }^{10}$.

Le Noir est aussi étiqueté à travers son langage, lequel ne réfère pas son être mais il est beaucoup plus marqué par la tentation de son modèle. Comme le 
reconnaît Archibald en affirmant: «Ah j'oubliais. Voleurs, nous avons tenté de dérober votre beau langage ${ }^{11}$.

Ainsi Les nègres se veulent un cri émanant d'une identité en mal de définition, de cette lutte inégale, le Noir semble prendre sa revanche sur la discrimination qu'il a longtemps subie, sur les multiples formes d'abus dont il était victime, et crie haut et fort contre son éternel retour au subalterne et à l'être soumis pour s'émanciper des carcans identitaires où l'enferme l'Autre.

Au terme de notre réflexion, nous constatons à quel point l'Afrique, de par son patrimoine et ses traditions ancestrales, de par le lien historique qui l'a unie à l'occident a incessamment constitué un espace fortement significatif et attractif pour lui. Le théâtre comme art du spectacle vivant qui interroge continuellement l'humain et qui lui permet d'extérioriser ses souffrances, d'exprimer ses aspirations et de dénoncer ses injustices n'a pas dérogé à cette règle. Nous pouvons même conclure que dans cette ouverture sur le constituant africain le théâtre occidental va rompre avec sa tendance naturaliste axée essentiellement sur la représentation du même pour verser dans un théâtre avant-gardiste qui redéfinit la théâtralité, recrée le spectaculaire en puisant dans ce que l'Afrique et l'Africain ont de particulier, d'humain et surtout d'universel.

\section{Notes}

1. Edward Gordon Craig, catalogue de l'exposition tenue à Manchester Art Gallery. Exhibition of Darwings and Models for hamlet, Macbeth, The Vikings and Other plays by Edward Gordon Craig, 1912, pp. 23-25.

2. Loc. cit.

3. Odette Aslan, "Le regard du masque », in Odette Aslan et Denis Bablet, Le masque: du rite au théâtre, de l'Équipe de recherches théâtrales du CNRS, Paris, éditions du CNRS, 1984, p. 10-22.

4. Jerzy Grotowski, Vers un théâtre pauvre, Lausanne, La Cité, Métropole, 1971, p. 31.

5. Jean Jaques Roubine, Théâtre et mise en scène 1880-1980, Paris, PUF, "Littératures modernes ", 1980, p. 48.

6. Antonin Artaud, Le Théâtre et son double, Paris, Gallimard, « Folio Essais »,1938, p. 14.

7. Guy Ossito Midiohouan, «Le théâtre négro-africain d'expression française depuis 1960 », Peuples Noirs Peuples Africains, n 31, 1983, p. 54-78.

8. J. Genet, Les Nègres, Paris, Gallimard, « Folio », 2005, p. 86.

9. Ibid., p. 60.

10. Ibid., p. 48.

11. Ibid., p. 86. 